\title{
Response probabilities for individual stimuli in
}

\section{learning tasks ${ }^{1}$}

ALLAN NASH, Florida Atlantic University, Boca Raton, Fla. 33432

Four squirrel monkeys were tested on a series of five learning problems. The first three problems involved the discrimination of two stimuli while each of the last two problems consisted of 10 stimuli divided into two response classes. Analysis of the proportion of correct responses to individual stimuli within a problem set revealed strong position-habit errors and suggested that $S$ does not learn the correct response to all stimuli of the same problem set with equal facility. This differential level of responding was more pronounced for the 10-stimulus problems than for the 2-stimulus problems.

Traditional methodology in discrimination learning studies has required $S$ to distinguish between two discriminanda. S's performance is usually analyzed as the proportion of trials on which a correct response is made. The present study used a specialized procedure in which a measure of correct responding could be obtained separately for each stimulus. In this way, it was possible to determine if S's performance reflects responding above chance to only one, or both, of the stimuli (Theios, 1968). The determination of response probabilities to individual stimuli may also apply to concept identification research when procedural limitations require the repeated use of the same stimulus set. To test the assumption that a general concept was acquired, it is necessary to show uniform changes in the probability of a correct response to each and every stimulus. Gradual increases in these probabilities or unusual variability in their asymptotic values would raise serious doubt that a concept was learned and would more likely suggest a simple conditioning of the response classes to specific stimuli as a result of the repeated presentations.

An earlier study of two-choice visual discrimination tasks (Nash \& Michels. 1966) had indicated that squirrel monkeys were particularly susceptible to position-habit errors during the early trials of a new discrimination task. Although Harlow (1959) discounts this source of error in traditional discrimination tasks, it was anticipated that it would be a major source of errors in squirrel monkeys if the task required $S$ to associate a positional response (right or left) with the different discriminanda when they were presented individually.

\section{METHOD}

The Ss consisted of four experimentally naive male squirrel monkeys, approximately 12-16 months old. The monkeys were adapted to the apparatus, which is described in detail elsewhere (Nash \& Michels, 1966). The transport cage was separated from the test chamber by two guillotine slides, one clear and one opaque, that could be independently raised or lowered. When the opaque slide was raised, $\mathrm{S}$ saw two illuminated doors with identical stimulus configurations on each. The stimulus configuration could be changed from trial to trial by inserting different pairs of stimulus slides in to the doors. After a 6-sec inspection period, the clear slide was also raised and $S$ could attempt to push open one of the two doors to retrieve an apple chip. A noncorrection procedure was used. In Problem Set 1, a training problem, the presence of the same random shape on each of the doors meant that the right door was unlocked on that trial. Two blank doors meant that the left door was unlocked. In all problem sets, the order of presenting the stimulus pairs was randomized over trials and each stimulus pair was presented an equal number of times. Initially, a sequential test of performance, STOP (Nash, 1969), was used to establish a criterion for terminating testing, but since most monkeys required several days of testing to learn the problems, the testing schedule was changed to a flat 50 trials per day with $66 \%$ correct $(p<.05)$ representing criterion for that block. Three (not necessarily consecutive) days of criterion performance were required before continuing to the next problem. Problem Set 2 was a two-stimulus discrimination problem. A horizontal rectangle on each door indicated a right-door response, vertical rectangles a left-door response. A right-door correct response in Problem Set 3 was associated with the presence of a black signal dot on each door located on the extreme right of

\section{Table 1}

Proportions of Correct Responses (Last 50 Trials) for Two-Stimuli Discrimination Problems

\begin{tabular}{|c|c|c|c|c|c|c|}
\hline \multirow{2}{*}{$\frac{\text { Monkey }}{1}$} & \multicolumn{2}{|c|}{$\begin{array}{l}\text { Problem Set } 1 \\
\text { Shape vs No Shape }\end{array}$} & \multicolumn{2}{|c|}{$\begin{array}{l}\text { Problem Set } 2 \\
\text { Horizontal vs Vertical }\end{array}$} & \multicolumn{2}{|c|}{$\begin{array}{c}\text { Problem Set } 3 \\
30^{\prime} \text { Clock vs } 6 \text { O'Clock }\end{array}$} \\
\hline & .86 & .75 & .85 & .61 & .83 & .67 \\
\hline 2 & .83 & .81 & $.82^{*}$ & $.55^{*}$ & .57 & .62 \\
\hline 3 & .73 & .83 & .58 & .73 & .69 & .58 \\
\hline 4 & .85 & .78 & .81 & .96 & .64 & .82 \\
\hline
\end{tabular}

an imaginary horizontal line that bisected the door into upper and lower halves ("3 o'clock" position). A left-door correct response was associated with the signal dot appearing at the bottom of an imaginary line bisecting each door into right and left halves ("6 o'clock" position). Problem Set 4 was a transition problem in that the correct door was also indicated by a signal dot in either the "3 o'clock" or "6 o'clock" position, but five stimulus pairs of each type were constructed and a second "distractor" dot was added at random to each pair. The position of this distractor dot was determined randomly, with the restriction that it be separated from the permanent signal dot by at least $1 \mathrm{~cm}$. Thus, Problem Set 4 consisted of 10 different pairs of stimulus slides with all five pairs of right-door-correct stimuli having the "3 o'clock" signal dot in common and all five pairs of left-door-correct stimuli having the "6 o'clock" signal dot in common. Problem Set 5 also consisted of 10 pairs of stimuli. A left-door-correct response for five of the pairs was always associated with a signal dot in the upper left corner of each door. A right-door-correct response for the other five pairs was always associated with a signal dot in the lower right corner of each door. A distractor dot was added in a randomly determined location to each of the 10 pairs of stimuli.

\section{RESULTS AND DISCUSSION}

The number of trials required to reach criterion on any given problem varied considerably (range: $200-1,700$ trials). For Problem Sets 1, 2, and 3, which consisted of two stimuli each, the proportions of correct responses to each of the two stimuli were computed for each $S$ over the last 50 trials. This measure defined the terminal probability of a correct response for $S$ on these problems, and these proportions are summarized in Table 1. Although the proportions vary widely between their limiting values of .5 (chance) and 1.00 , the power of such tests is low; the only statistically significant difference at the .05 level is for Monkey 2 on Problem Set 2 .

In Problem Sets 4 and 5, each of the 10 stimuli was presented only five times in a block of 50 trials. Therefore, to achieve more stable estimates of the correct 


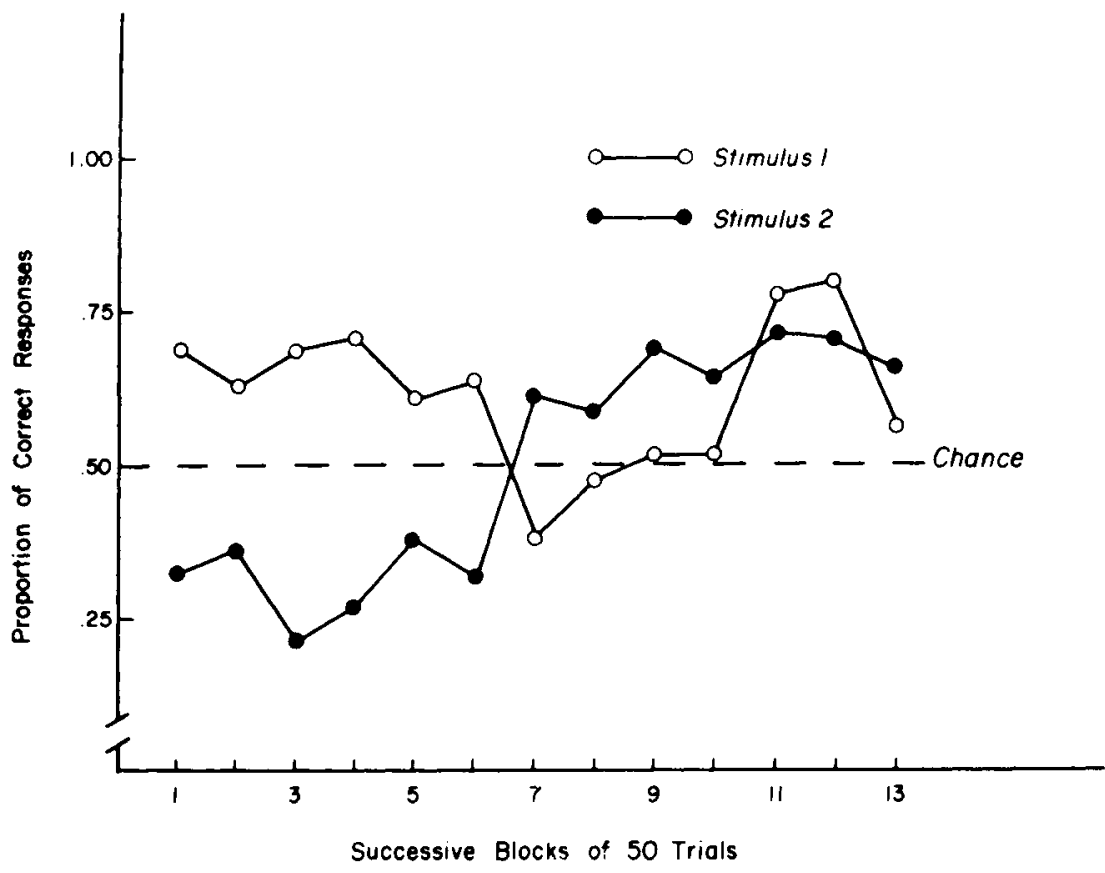

response probabilities, the proportions of correct responses to each stimulus were computed over the last 150 trials. These data are presented in Table 2. Statistical comparisons among each of the 10 stimuli in Problem Sets 4 and 5 are difficult to accomplish on an individual $S$ basis, although this approach would be most meaningful. On a purely descriptive level, these proportions are observed to vary widely over the 10 stimuli for any given $S$. Monkey 1 on Problem Set 5, for example, correctly responded to .95 of the presentations of Stimulus 10, but only got .38 of the trials involving Stimulus 9 correct. While there appears to be some consistency between Ss regarding which

stimuli were difficult and which were easy, analysis of the geometric properties of the stimuli revealed no obvious relationships.

Figure 1 shows the proportions of correct responses of one monkey to the two stimuli of Problem Set 2. The nearly perfect symmetry of the proportions around the chance line for the first few blocks of trials was typical and indicates a positional response bias. Since S's responses were right or left directed, he could at one extreme be correct $100 \%$ of the time for one stimulus and $0 \%$ of the time for the other simply by responding to the same side on all trials.

This positional preference phenomenon appeared during the early trials for all Ss

Table 2

Proportions of Correct Responses (Last 150 Trials) for Ten-Stimuli Discrimination Problems

\begin{tabular}{|c|c|c|c|c|c|c|c|c|c|c|}
\hline \multirow{2}{*}{$\begin{array}{l}\overrightarrow{0} \\
\stackrel{0}{E} \\
\stackrel{0}{0} \\
\sum\end{array}$} & \multicolumn{10}{|c|}{$\begin{array}{l}\text { Problem Set } 4 \text { (3 O'Clock vs } 60^{\prime} \text { Clock with Distractors) } \\
3 \text { O'Clock Stimuli }\end{array}$} \\
\hline & 1 & 2 & 3 & 4 & 5 & 6 & 7 & 8 & 9 & 10 \\
\hline 1 & .60 & .53 & .87 & .80 & .93 & .73 & .80 & .87 & .73 & .73 \\
\hline 2 & .40 & .53 & .53 & .60 & .71 & .85 & .87 & 1.00 & .76 & .87 \\
\hline 3 & .57 & .73 & .73 & .73 & .53 & .73 & .60 & .72 & .88 & .68 \\
\hline 4 & .60 & .73 & .80 & .63 & .93 & .67 & .27 & .87 & .77 & .47 \\
\hline
\end{tabular}

Problem Set 5 (Upper Left vs Lower Right with Distractors)

\begin{tabular}{|c|c|c|c|c|c|c|c|c|c|}
\hline & Upper & Left & Stimuli & & & & Right & unli & \\
\hline 1 & 2 & 3 & 4 & 5 & 6 & 7 & 8 & 9 & 10 \\
\hline .67 & .85 & .75 & .72 & .62 & .66 & .92 & .87 & .38 & .95 \\
\hline .93 & .73 & .87 & .81 & .60 & .73 & .87 & 1.00 & .78 & .80 \\
\hline .46 & .53 & .87 & .92 & .47 & .67 & .81 & .60 & .60 & .60 \\
\hline .67 & .53 & .60 & .63 & .33 & .67 & .87 & .47 & .30 & .73 \\
\hline
\end{tabular}

Fig. 1. Proportions of correct responses to individual stimuli for one monkey on Problem Set 2.

on the majority of the problem sets. Contrary to Harlow's (1959) contention that position habit errors are essentially an unimportant source of error for primates in discrimination learning studies, the present work suggests that they are a major source of error among lower primates when the response to be learned is itself one of position. With the increased use of automated data collection, it is quite easy to miss such properties of the data unless analyses of responses to individual stimuli are routinely carried out. Notice in Fig. 1 that the traditionally computed proportion of correct responses, pooling responses over both stimuli, would have produced data points over the first seven blocks that fell very close to the chance line. Since this agrees with our intuition that, prior to learning. performance should be neas the chance level, we would not be likely to question such data, and yet we see that the nature of S's task in this study has produced specific response patterns that individually deviate most markedly from chance.

The observed variability among the terminal proportions of correct responses suggests that squirrel monkeys learn the individual responses in tasks of this type with varying degrees of success. The analysis of correct responses to individual stimuli also reveals response patterns such as position habit errors that would be otherwise obscured by the more traditional measure that sums over all stimuli.

\section{REFERENCES}

HARLOW, H. F. Learning set and error factor theory. In S. Koch (Ed.), Psychology: A study of a science. Vol. 2. New York: McGraw-Hill, 1959. Pp. 492-537.

NASH, A. A sequential test of performance (STOP) for all-or-none learning data. Psychonomic Science, 1969, 14, 77-79.

NASH, A. \& MICHELS, K. M. Squirrel monkeys and discrimination learning: Figural interactions, redundancies, and random shapes. Journal of Experimental Psychology, 1966, 72, 132-137.

THEIOS, J. Finite integer models for learning in individual subjects. Psychological Review, $1968,75,292-307$. NOTES

1. This research was supported in part by Research Grant No. HD-1454 from the National Institute of Child Health and Human Development. 